\title{
SYNTHETIC APERTURE RADAR AUTOFOCUS VIA SEMIDEFINITE RELAXATION
}

\author{
Kuang-Hung Liu*, Student Member, IEEE, Ami Wiesel ${ }^{\dagger}$, Member, IEEE, \\ and David C. Munson, Jr.*, Fellow, IEEE,
}

\begin{abstract}
The autofocus problem in Synthetic Aperture Radar imaging amounts to estimating unknown phase errors caused by unknown platform or target motion, or a time- or spatiallyvarying transmission medium. At the heart of three state-ofthe-art autofocus algorithms, namely Phase Gradient Autofocus, Multichannel Autofocus (MCA) and Fourier-domain Multichannel Autofocus (FMCA), is the solution of a constant modulus quadratic program (CMQP). Currently, these algorithms solve CMQP by using an eigenvalue relaxation approach. We propose an alternative relaxation approach based on semidefinite programming, which has recently attracted considerable attention in other signal processing problems. Experimental results show that our proposed methods provide promising performance improvements for MCA and FMCA through an increase in computational complexity.
\end{abstract}

Index Terms-Synthetic aperture radar, Autofocus, Multichannel Autofocus, Fourier-domain Multichannel Autofocus, Semidefinite relaxation.

\section{INTRODUCTION}

Synthetic aperture radar (SAR) offers a means of producing high-resolution microwave images using an antenna of small size. High resolution in the range direction is achieved through traditional pulse compression, while high resolution in the cross-range direction is obtained by illuminating the target from many look angles. In one form of SAR, termed spotlight mode, the radar antenna is continuously steered to illuminate the target with each transmitted microwave pulse. When the same antenna is used for both transmitting and receiving, it is referred to as monostatic SAR, while in bistatic SAR separate antennas are used for transmitting and receiving. The collected returned signal in spotlight mode SAR can be conveniently modeled using a tomographic formulation, which allows us to view returned signals as data lying in the Fourier domain of the target reflectivity, after quadrature demodulation [1], [2]. The Fourier data lies on a polar annulus with parameters determined by the radar waveform and the radar's range of look angles. The SAR image customarily is produced using polar-to-Cartesian interpolation followed by 2-D Fourier inversion. However, due to unknown signal delays resulting from

* The authors are with the Department of Electrical Engineering and Computer Science, University of Michigan, Ann Arbor, MI 48109-2122 USA (e-mail: khliu@umich.edu, munson@umich.edu).

$\dagger$ The author is with The Rachel and Selim Benin School of Computer Science and Engineering at the Hebrew University of Jerusalem, Israel. The work of A. Wiesel was supported by the Marie Curie Outgoing International Fellowship within the 7th European Community Framework Programme. (email: amiw@cs.huji.ac.il)

This work was also partially supported by Air Force Office of Scientific Research grant No. FA9550-06-1-0324. inaccurate range measurements or signal propagation effects, there will be demodulation timing errors at the radar receiver [1]. Even though motion compensation systems have improved (using GPS), those systems do not help with phase errors introduced by perturbations in the ionosphere in spaceborne SAR imaging. Timing errors at the demodulator will produce unknown phase errors in the collected Fourier data, which can cause the reconstructed image to suffer distortion. Autofocus algorithms apply signal processing techniques to remove the undesired phase errors and restore the focused image.

Most existing autofocus algorithms invoke assumptions on the properties of the unknown phase function or characteristics of the underlying SAR scene. An early class of autofocus algorithms assumes that the phase error function can be described by a finite polynomial expansion [3], [4]. Other autofocus techniques estimate the phase errors by maximizing the sharpness of the reconstructed image [5], [6]. Some popular metrics that measure the image sharpness include entropy and various powers of the image intensity [7]. The widely used Phase Gradient Autofocus (PGA) technique is conceptually based on an image model where each range coordinate has a single point target embedded in white complex Gaussian clutter, and applies maximum-likelihood (ML) phase estimation [8]. The Multichannel Autofocus (MCA) algorithm assumes a known region in the SAR image has pixels with zero or nearlyzero value [9]. Such a region can be identified within the sidelobes of the antenna pattern. Recently, the Fourier-domain Multichannel Autofocus (FMCA) algorithm was devised to allow for a wider range of look angles than permitted by other techniques [10]. FMCA has been shown to provide superior image restoration capability as compared to other autofocus methods.

At the core of the three state-of-the-art autofocus algorithms, PGA, MCA and FMCA is the solution to a Constant Modulus Quadratic Program (CMQP) of the following form

$$
\begin{aligned}
\min _{\mathbf{x} \in \mathbb{C}^{M}} & \mathbf{x}^{H} \mathbf{Q x} \\
\text { s.t } & \left|\mathbf{x}_{i}\right|=1, i=0, \ldots, M-1 .
\end{aligned}
$$

This problem is known to be NP-hard; thus, the best we can hope for is an approximation. All three algorithms use eigenvalue relaxation to approximate the original CMQP. In this paper, we propose an alternative approximation based on modern conic optimization known as semidefinite relaxation (SDR). SDR offers a compromise. It is known to provide a more accurate approximation of the CMQP at the cost of 
complicating the underlying optimization problem.

SDR recently has been applied to many problems in communications and signal processing [11], [12], [13], [14]. Basically, SDR approximates a quadratic problem with a convex optimization problem by first lifting the problem to a higher dimension and then relaxing the nonconvex constraints. To the best of our knowledge, this is the first time that SDR has been applied to the problem of SAR autofocus. This problem formulation is similar to the discrete symbol detection problem in communication systems, which has recently gained considerable attention [12]. However, the feasible set is a continuous constant modulus set as described in (1) above, and is not discrete. Some theoretical results on the performance of SDR for the continuous symbol case are given in [15], [16], [17]. Our simulation results suggest that, combined with MCA and FMCA, SDR is a promising autofocus technique. We note that currently solving SDR requires polynomial time and may not be amenable to online processing. Nonetheless, there may be crucial situations where it is imperative that an image be focused as well as possible, using computationally intensive offline processing.

This paper expands upon a preliminary version of our work reported in [18]. The organization of the paper is as follows. We will briefly review the concept of SDR in Section II. Section III presents the problem formulation for PGA, MCA and FMCA and also discusses how we can improve these existing techniques by using SDR. Simulation results are given in Section IV. Finally, we summarize our work in Section V.

In this paper, a superscript $(\cdot)^{H}$ denotes the hermitian transpose and $(\cdot)^{T}$ denotes transpose. The function $\operatorname{vec}(\mathbf{A})$ stacks the columns of matrix $\mathbf{A}$ to produce a column vector. The function $\operatorname{Diag}(\mathbf{u})$ forms a diagonal matrix with vector $\mathbf{u}$ on the main diagonal. For a complex-valued vector $\mathbf{a}$, the function $\angle(\mathbf{a})$ retains the angular part of $\mathbf{a}$. We write $\mathbf{A} \succeq 0$ to indicate that $\mathbf{A}$ is a positive semidefinite matrix, and let $\operatorname{tr}(\mathbf{A})$ denote the trace of $\mathbf{A}$.

\section{REVIEW OF SEMIDEFINITE RELAXATION}

In this section, we briefly review the topic of semidefinite programming (SDP) and its application to approximate nonconvex CMQP problems. More details on SDP can be found in [19], [20]. The SDR approximation is described in [13].

\section{A. Semidefinite Programming}

In recent years, there has been considerable progress on the development of efficient algorithms for solving a variety of optimization problems. In particular, significant attention has been devoted to SDP, a generalization of classical linear programming to include linear matrix inequalities. The standard form of an SDP is [19], [20]:

$$
\begin{array}{ll}
\min & \operatorname{tr}(\mathbf{Q X}) \\
\text { s.t. } & \operatorname{tr}\left(\mathbf{A}_{i} \mathbf{X}\right)=b_{i}, i=0, \ldots, M-1 \\
& \mathbf{X} \succeq 0,
\end{array}
$$

where $\mathbf{Q}, \mathbf{A}_{i}, i=0, \ldots, M-1$ are the data parameters and $\mathbf{X}$ is the optimization variable. SDP belongs to a class of problems knowns as conic optimization problems whose global optimum can be efficiently found using standard algorithms.

The most promising technique at present for solving smallto-medium-scale SDP is the interior-point (IP) method. There exist off-the-shelf software packages based on IP for solving general SDP [21]. In many problems, it is more computationally efficient to use a customized IP method tailored to the problem of interest (e.g., [12]). Unfortunately, IP methods are not appropriate for large-scale problems, such as the ones addressed in this paper, because the memory and computational costs of even one IP iteration are too high. In such scenarios, first-order methods, with simple iterations, must be utilized. A classical method is the spectral bundle method developed in [22], [23]. The standard SDP involves real parameters and variables, but can be easily generalized to account for complex values through a change of variables (e.g., [12]), or specifically tailored complex optimization methods.

\section{B. Constant Modulus Quadratic Programming}

One promising application of SDP is in the approximation of the complex Constant Modulus Quadratic Programming (CMQP) problem [13]. In particular, CMQP can be written as

$$
\begin{aligned}
(C M Q P) \quad \min _{\mathbf{x} \in \mathbb{C}^{M}} & \mathbf{x}^{H} \mathbf{Q} \mathbf{x} \\
\text { s.t } & \left|\mathbf{x}_{i}\right|=1, i=0, \ldots, M-1 .
\end{aligned}
$$

It is known that CMQP is NP-hard [16], and thus, for large problem sizes, the best we can hope for is an approximation algorithm.

A natural approximation to $\mathrm{CMQP}$ is eigenvalue relaxation (EVR), which can be written as

$$
\begin{aligned}
(E V R) & \min _{\mathbf{x} \in \mathbb{C}^{M}} \mathbf{x}^{H} \mathbf{Q} \mathbf{x} \\
\text { s.t } & \mathbf{x}^{H} \mathbf{x}=M .
\end{aligned}
$$

The main advantage of EVR is that the problem $(E V R)$ has a simple closed-form solution. Using the variational characterization of singular values, the optimal solution to EVR is the right singular vector of $\mathbf{Q}$ that corresponds to the minimum singular value. Clearly, if this eigenvector satisfies the original $(C M Q P)$ constraints, then it is the optimal solution to $(C M Q P)$ as well and the relaxation is tight. Otherwise, we can obtain an approximate solution $\tilde{\mathbf{x}}$ by rounding the minimum right singular vector, denoted by $\mathbf{v}$, as

$$
\tilde{\mathbf{x}}=e^{j \angle(\mathbf{v})} \text {. }
$$

Recently, a more advanced relaxation scheme, SDR, has been proposed. This relaxation can be derived through Lagrange duality or via a lift-and-relax argument (see exercise 5.39 in [19]). For completeness, we review the latter derivation. Using the identity $\mathbf{x}^{H} \mathbf{Q} \mathbf{x}=\operatorname{tr}\left(\mathbf{Q} \mathbf{x} \mathbf{x}^{H}\right)$, we first 
lift the solution space of $(C M Q P)$ from vectors to positive semidefinite matrices to obtain

$$
\begin{array}{cl}
\left(C M Q P^{\prime}\right) \quad \min _{\mathbf{X} \in \mathbb{C}^{M \times M}} & \operatorname{tr}(\mathbf{Q X}) \\
\text { s.t } & \mathbf{X}_{i i}=1, i=0, \ldots, M-1 \\
& \mathbf{X} \succeq 0 \\
& \operatorname{rank}(\mathbf{X})=1 .
\end{array}
$$

Problems $(C M Q P)$ and $\left(C M Q P^{\prime}\right)$ are equivalent since the solution to $\left(C M Q P^{\prime}\right), \mathbf{X}$, can be expressed as $\mathbf{X}=\mathbf{x x}^{H}$ with $\left|\mathbf{x}_{i}\right|=1, i=0, \ldots, M-1$. Problem $\left(C M Q P^{\prime}\right)$ has a nonconvex feasible set due to the rank 1 constraint and cannot be solved efficiently. Instead, we relax the feasible set of $\left(C M Q P^{\prime}\right)$ to obtain the revised problem $(S D R)$ :

$(S D R)$

$$
\begin{array}{cl}
\min _{\mathbf{X} \in \mathbb{C}^{M \times M}} & \operatorname{tr}(\mathbf{Q X}) \\
\text { s.t } & \mathbf{X}_{i i}=1, i=0, \ldots, M-1 \\
& \mathbf{X} \succeq 0 .
\end{array}
$$

The above optimization problem is a relaxation of $\left(C M Q P^{\prime}\right)$ and is a SDP with $\mathbf{A}_{i}=\operatorname{Diag}\left(\mathbf{e}_{i}\right)$ where $\mathbf{e}_{i}$ is the $i$ th column of the identity matrix and $b_{i}=1$ for all $i$. Thus, it can be efficiently solved as explained above.

Just like the EVR approach, the SDR must be complemented with an additional rounding scheme which uses its solution to generate an approximate feasible solution to $(C M Q P)$. Let $\hat{\mathbf{X}}$ be the solution of $(S D R)$. If $\operatorname{rank}(\hat{\mathbf{X}})=1$, then $\hat{\mathbf{X}}=\hat{\mathbf{x}} \hat{\mathbf{x}}^{H}$ is an optimal solution to $(C M Q P)$, and the $(C M Q P)$ problem is solved exactly. Otherwise, we can use $\hat{\mathbf{X}}$ to obtain a feasible approximate solution to $(C M Q P)$. There are several methods we might employ. Here we focus on the randomization method [16]. Let $\hat{\mathbf{X}}=\hat{\mathbf{V}} \hat{\mathbf{V}}^{H}$ where $\hat{\mathbf{V}}=\left[\hat{\mathbf{v}}_{1}, \ldots, \hat{\mathbf{v}}_{n}\right]$ is a square-root factor of $\hat{\mathbf{X}}$. Because we relax the rank-1 constraint for $\hat{\mathbf{X}}, n$ may be greater than 1 . The randomization method generates $M_{\text {rand }}$ complex gaussian vectors $\mathbf{u}_{1}, \mathbf{u}_{2}, \ldots, \mathbf{u}_{M_{\text {rand }}}$ that are independent with zero mean and covariance I. It then computes $\mathbf{y}_{i}=\angle\left(\hat{\mathbf{V}} \mathbf{u}_{i}\right), i=1, \ldots, M_{\text {rand }}$ and approximates a feasible solution, $\tilde{\mathbf{x}}$, to $(C M Q P)$ as

$$
\tilde{\mathbf{x}}=\underset{\mathbf{y}_{1}, \ldots, \mathbf{y}_{M_{\text {rand }}}}{\operatorname{argmin}} \mathbf{y}_{i}^{H} \mathbf{Q} \mathbf{y}_{i} .
$$

\section{Approximation quality}

We now discuss the approximation quality of SDR compared with that of EVR. Our main result is summarized in Theorem 1.

Theorem 1: Let $v_{o}^{*}, v_{s d r}^{*}$ and $v_{e v r}^{*}$ represent the optimal objective function values found for problems $(C M Q P),(S D R)$ and $(E V R)$, respectively. Then

$$
v_{o}^{*} \geq v_{s d r}^{*} \geq v_{e v r}^{*} .
$$

Proof: First, we have shown that $(S D R)$ is a relaxation of $\left(C M Q P^{\prime}\right)$ and $\left(C M Q P^{\prime}\right)$ is equivalent to $(C M Q P)$. This immediately gives us

$$
v_{o}^{*} \geq v_{s d r}^{*}
$$

To show the second inequality, we note that $(E V R)$ is equivalent to the following problem:

$$
\left(E V R^{\prime}\right)
$$

$$
\begin{array}{ll}
\min _{\mathbf{X} \in \mathbb{C}^{M \times M}} & \operatorname{tr}(\mathbf{Q X}) \\
\text { s.t } & \operatorname{tr}(\mathbf{X})=M \\
& \mathbf{X} \succeq 0 .
\end{array}
$$

This is easily proved by showing that $\left(E V R^{\prime}\right)$ is both a lower bound and an upper bound for $(E V R)$; thus it is tight. Let $v_{e v r^{\prime}}^{*}$ be the optimal objective function value found for $\left(E V R^{\prime}\right)$ and we have $v_{e v r^{\prime}}^{*}=v_{e v r}^{*}$. On the other hand, $\left(E V R^{\prime}\right)$ can be viewed as a relaxation of $(S D R)$ by relaxing the $\mathbf{X}_{i i}=1, \forall i$ constraint to $\operatorname{tr}(\mathbf{X})=M$, and therefore

$$
v_{s d r}^{*} \geq v_{e v r^{\prime}}^{*}=v_{e v r}^{*} .
$$

Theorem 1 shows that in terms of objective function value, SDR is a tighter relaxation than the natural EVR approach. However, it is important to emphasize that these results do not provide any guarantees on the quality of the solution itself.

Another result, due to So et al. [16], provides quality assurance for approximating a certain form of CMQP using SDR. Their result states that SDR plus randomization is a $\frac{\pi}{4}$-approximation algorithm for CMQP with $\mathbf{Q}$ in (3) that is negative semidefinite, i.e.,

$$
v_{p}^{*} \geq \mathrm{E}\left[\mathbf{y}^{H} \mathbf{Q y}\right] \geq \frac{\pi}{4} v_{p}^{*},
$$

where $\mathbf{y}=\angle(\hat{\mathbf{V}} \mathbf{u})$ and $\mathbf{u}$ is a normally distributed complex vector.

\section{Autofocus In Synthetic Aperture Radar}

In this section we briefly review the tomographic formulation of spotlight-mode SAR for both the monostatic and bistatic cases and refer the reader to [1] for more details. Next, we formulate the autofocus problem solution using the PGA, MCA and FMCA approaches. The main message of this section is that all of these approaches lead to a CMQP problem. Previous work has approximated this problem using the EVR approach, and we propose to enhance the performance using the SDR technique described above.

A spotlight-mode SAR system can be mathematically described using the following tomographic model. A target scene, represented by a complex-valued reflectivity function $g(x, y)$, is illuminated by SAR over the radar's range of look angles, $\theta_{0}, \cdots, \theta_{M-1}$. The discrete signal collected from look angle $\theta_{m}$ can be modeled as a "slice" of the 2-D Fourier transform of $g(x, y)$, taken at angle $\theta_{m}$ and sampled at radiuses $R_{0}, \cdots, R_{N-1}$. These signals are conveniently collected into an $M \times N$ matrix $\mathbf{G}_{p}$, known as the polar-format Fourier transform of $g(x, y)$. 
SAR image reconstruction estimates the amplitudes of the target scene using the polar-format Fourier data. The classical image formation technique interpolates $\mathbf{G}_{p}$ onto a Cartesian grid and then applies an inverse 2-D discrete Fourier transform. The result is denoted by $\hat{g}\left(x_{i}, y_{i}\right)$ for $i=1, \cdots, I$. Under suitable conditions, it has been demonstrated that the speckle image $\left|\hat{g}\left(x_{i}, y_{i}\right)\right|$ can be a good approximation for the amplitudes $\left|g\left(x_{i}, y_{i}\right)\right|$, so long as the phases, $\angle g\left(x_{i}, y_{i}\right)$, are approximately uncorrelated [24].

A challenge in SAR imaging is that in order to correctly demodulate the returned signal, the two-way travel time of the transmitted signal must be known. In practice, due to unknown signal delays resulting from inaccurate range measurements or signal propagation effects, the polar-format Fourier data is contaminated with unknown phase errors that cause the reconstructed image to suffer distortion. The measurements at a given look angle suffer from the same unknown delay, and, under a narrow-band assumption, their unknown phase is constant. The delays, and their associated phases, change between different look angles. This results in the error model

$$
\tilde{\mathbf{G}}_{p}[m, n]=\mathbf{G}_{p}[m, n] e^{j \phi(m)}+\mathbf{W}[m, n],
$$

where $\phi(m) \in \mathbb{R}, m=0,1, \ldots, M-1$ are unknown phases, and $\mathbf{W}[m, n]$ represents additional noise [1].

SAR reconstruction amounts to estimating the speckle image $\left|\hat{g}\left(x_{i}, y_{i}\right)\right|$ given the observations $\tilde{\mathbf{G}}_{p}$. Note that even without unknown phases, SAR reconstruction produces a speckle image that is similar to $\left|g\left(x_{i}, y_{i}\right)\right|$ but not identical. Moreover, as the additive noise increases, the quality of the speckle image degrades. The goal of SAR autofocus is to recover this speckle image (and not the true reflectivity function) in the presence of unknown phases. In this paper, we will address this problem using a natural approach that first estimates the unknown phases, compensates for them and finally reconstructs the speckle image using classical techniques.

\section{A. Phase Gradient Autofocus}

PGA is the autofocus method most widely employed in practice. It is motivated by considering a scenario where each row of $g$ contains only a single point reflector located at the center of the row. These reflectors are modeled as mutually independent and identically distributed (i.i.d) zeromean complex Gaussian random variables with variance $\sigma_{a}^{2}$, whereas the surrounding clutter is represented by i.i.d. zeromean complex Gaussian random variables with variance $\sigma_{n}^{2}$. Let $\tilde{\mathbf{G}}_{r c}$ denote the range-compressed data defined as $\tilde{\mathbf{G}}_{p}$ after undergoing a 1-D inverse Fourier transform in the $n$ dimension. Then, the rows of $\tilde{\mathbf{G}}_{r c}$ are i.i.d. realizations of a zero mean complex Gaussian vector with covariance

$$
\sigma_{a}^{2} \mathbf{x} \mathbf{x}^{H}+\sigma_{n}^{2} \mathbf{I}
$$

where $\mathbf{x}=e^{j \phi}$ is the phase vector $(\phi=$ $\left.[\phi(0), \phi(1), \cdots, \phi(M-1)]^{T}\right)$ which satisfies $\left|\mathbf{x}_{i}\right|=1$ for $i=0,1, \cdots, M-1$.

PGA estimates $\mathbf{x}$ using a maximum likelihood approach. After simple algebraic manipulations, the problem reduces to

$$
(P-P G A) \quad e^{j \hat{\boldsymbol{\phi}}_{P G A}}=\underset{\mathbf{x}:\left|\mathbf{x}_{i}\right|=1}{\operatorname{argmax}} \mathbf{x}^{H} \tilde{\mathbf{G}}_{r c}^{T}\left(\tilde{\mathbf{G}}_{r c}^{T}\right)^{H} \mathbf{x},
$$

It is easy to see that $(P-P G A)$ is a CMQP with $\mathbf{Q}=$ $-\tilde{\mathbf{G}}_{r c}^{T}\left(\tilde{\mathbf{G}}_{r c}^{T}\right)^{H}$. It was shown in [1] that, under the assumed single point reflector model and at high signal-to-noise ratio, the eigenvalues of $\mathrm{E}[\mathbf{Q}]$ have only one dominating term and its corresponding eigenvector is the true autofocus phase error vector (with unknown time shift). In other words, $(P-P G A)$ satisfies the following

$$
v_{o}^{*} \approx v_{e v r}^{*}
$$

and EVR is tight.

For completeness, we note that the above scheme is not the full PGA algorithm, but is its core step. The full algorithm is an iterative technique where at each iteration the algorithm first preprocesses the obtained phase-compensated image so that it can be more accurately described by the assumed point target model. Then the phase errors are estimated by using (16) and a refined image is constructed. For a complete description of the full PGA algorithm, see [1]. Also, note that in practice a simpler PGA algorithm is commonly used where the phase difference between adjacent pulses is estimated. This method can be shown to be a special case of the maximum-likelihood technique presented above.

\section{B. Multichannel Autofocus}

An alternative image model was recently proposed by Morrison et al. [9]. The autofocus algorithm they developed is called Multichannel Autofocus (MCA). The MCA reconstruction method assumes that there is a known region in the image that consists of nearly zero-valued pixels, i.e., $g\left(x_{r}, y_{r}\right) \approx 0$ for $r=0, \cdots, R-1$. This prior knowledge can be inferred by using the low-return region of the antenna pattern [9]. Using this knowledge and "reverse engineering," MCA searches for the phases that will result in a reconstructed image with

$$
\left|\hat{g}\left(x_{r}, y_{r}\right)\right| \approx 0 \quad \text { for } \quad r=0, \cdots, R-1 .
$$

For simplicity, MCA assumes that the range of look angles is small enough so that $\mathbf{G}_{p}$ can be well approximated by a Cartesian grid. Therefore, the polar-to-Cartesian interpolation process can be ignored in the image reconstruction process and (18) reduces to solving

$$
\frac{1}{M N} \sum_{m, n} \tilde{\mathbf{G}}_{p}[m, n] e^{-j \phi(m)} e^{j 2 \pi\left(\frac{x_{r} m}{K}+\frac{y_{r} n}{L}\right)} \approx 0
$$

for $\phi(0), \phi(1), \cdots, \phi(M-1)$ and for $r=0,1, \ldots, R-1$. Using vector notation, the autofocus problem reduces to finding a vector $\mathbf{x} \in \mathbb{C}^{M}$ such that

$$
\mathbf{A x}=0, \quad\left|\mathbf{x}_{i}\right|=1, \quad i=0,1, \cdots, M-1,
$$

where $\mathbf{A}$ is an $R$ by $M$ matrix with elements

$$
\mathbf{A}[m, r]=\frac{1}{M N} \sum_{n} \tilde{\mathbf{G}}_{p}[m, n] e^{j 2 \pi\left(\frac{x_{r} m}{M}+\frac{y_{r} n}{N}\right)} .
$$

A naive approach to this problem is to try solving this system of equations exactly. In practice, the measurements are noisy and the approximately zero-valued pixels are not exactly zero. 
Instead, requiring that the low-return region has minimum energy leads to the following optimization problem:

$$
(P-M C A) \quad e^{j \hat{\boldsymbol{\phi}}_{M C A}}=\underset{\mathbf{x}:\left|\mathbf{x}_{i}\right|=1}{\operatorname{argmin}}\|\mathbf{A} \mathbf{x}\|^{2} .
$$

Thus, the MCA approach reduces to solving a standard CMQP with $\mathbf{Q}=\mathbf{A}^{H} \mathbf{A}$. In the original MCA paper [9], an approximate solution was proposed using an EVR approach. A better approximation can be obtained by using our proposed SDR at the cost of increased computational complexity.

For completeness, we note that the original MCA derivation used a more general framework with arbitrary basis functions, and worked in the spatial domain rather than the Fourier domain. The SDR method can be applied equally well in that framework.

\section{Fourier-domain Multichannel Autofocus}

Only on rare occasions does SAR operate over a range of look angles spanning a small fraction of one degree. MCA breaks down quickly as the range of look angles becomes larger [25]. FMCA is a generalization of MCA that recognizes that the collected Fourier data is in polar format and the interpolation process cannot be ignored. FMCA requires that the polar-to-Cartesian interpolation be linear to preserve the linear structure of the inverse problem. In practice, linear interpolation is almost always used, for example see [1]. With linear interpolation, the Cartesian grided data, denoted by $\mathbf{G}_{c}[k, l]$, can be expressed as.

$$
\mathbf{G}_{c}[k, l]=\sum_{m, n} \alpha(k, l, m, n) \mathbf{G}_{p}[m, n],
$$

where $\alpha(k, l, m, n)$ are the interpolation coefficients. In this paper we used a nearest-neighbor interpolation for simplicity. Thus $\alpha(k, l, m, n)=1$ when $\mathbf{G}_{p}[m, n]$ is closest to $\mathbf{G}_{c}[k, l]$ and 0 otherwise. In principle, any linear interpolation can be used and accommodated within this framework. Assuming $g\left(x_{r}, y_{r}\right)$ are nearly zero, and together with (23), (19) and (14), FMCA formulates the autofocus problem as the solution to

$\frac{1}{K L} \sum_{k, l} \sum_{n, m} \alpha(k, l, m, n) \tilde{\mathbf{G}}_{p}[m, n] e^{-j \phi(m)} e^{j 2 \pi\left(\frac{x_{r} k}{K}+\frac{y_{r} l}{L}\right)} \approx 0$

for all $r=0,1, \ldots, R-1$. Similar to MCA, the FMCA problem reduces to finding a vector $\mathbf{x}$ such that

$$
\mathbf{B x}=0, \quad\left|\mathbf{x}_{i}\right|=1, \quad i=0,1, \cdots, M-1
$$

where $\mathrm{B}$ is an $R$ by $M$ matrix with elements

$$
\begin{aligned}
\mathbf{B}[m, r]= & \frac{1}{K L} \sum_{k, l}\left(\sum_{n} \alpha(k, l, m, n) \tilde{\mathbf{G}}_{p}[m, n]\right) \\
& e^{j 2 \pi\left(\frac{x_{r} k}{K}+\frac{y_{r l} l}{L}\right)} .
\end{aligned}
$$

In practice, it is more reasonable to assume that the low-return region has small energy. Therefore, FMCA attempts to solve the following optimization problem:

$$
(P-F M C A) \quad e^{j \hat{\boldsymbol{\phi}}_{F M C A}}=\underset{\mathbf{x}:\left|\mathbf{x}_{i}\right|=1}{\operatorname{argmin}}\|\mathbf{B} \mathbf{x}\|^{2} .
$$

Thus the FMCA approach reduces to solving a standard CMQP with $\mathbf{Q}=\mathbf{B}^{H} \mathbf{B}$. In the original FMCA method [10], an approximate solution was proposed using an EVR approach. In the next section, we will demonstrate that a better approximation can be obtained using the SDR technique in exchange for an increase in computational complexity.

\section{Simulation Results}

In this section, we provide a few illustrative experiments showing the advantages of the SDR approach in comparison to existing autofocus algorithms. We examine narrow and wide ranges of look angles, as well as the bistatic scenario. In this section we denote MCA-SDR and FMCA-SDR as the algorithms using SDR to approximate $(P-M C A)$ in (22) and $(P-F M C A)$ in (27), respectively.

\section{A. SAR Simulator}

In order to test the different algorithms, we built a SAR simulator which used a SAR image, $g$, to generate Fourier observations $\tilde{\mathbf{G}}_{p}$. The complex images, $g$, we used were taken from the public SAR database provided by Sandia National Laboratory [26]. The complex image was multiplied by a 2-D antenna pattern, and then a 2-D discrete Fourier transform (DFT) was applied. The resulting Fourier matrix was then linearly interpolated to a polar grid to obtain the $M \times N$ polar format data $\mathbf{G}_{p}$ where $M$ and $N$ denote the number of samples in the range and cross-range directions, respectively. The shape of the polar grid was dominated by the range of viewing angles adopted in each simulation. Finally, the noisy and phase-corrupted observations $\tilde{\mathbf{G}}_{p}$ were simulated as expressed in (14). Our simulator generated a white phase error where each $\phi(m)$ was independent and uniformly distributed between $-\pi$ and $\pi$. This is considered the most challenging type of phase error function. The additive noise was independent, zero-mean complex Gaussian with variance $\sigma_{n}^{2}$ determined by the signal-to-noise ratio (SNR). Here, the SNR is defined as $\mathrm{SNR}=20 \log _{10}\left\{\left(\sum_{m, n}\left|\tilde{\mathbf{G}}_{p}[n, m]\right| / N M\right) / \sigma_{n}\right\}$.

Our simulator employed two types of antenna patterns: a trapezoidal pattern with unit gain over 90 percent of the image and linearly increasing attenuation to $\gamma, 0<\gamma<1$, at the edge of the image; and a more realistic 2-D sinc-squared antenna pattern with 95 percent of the mainlobe covering the image.

\section{B. Reconstruction Experiments}

In our first set of simulations we adopted a SAR system operating across a narrow range of look angles, so that the collected polar Fourier data was nearly Cartesian. In this simulation we let $M=N=256$. We compared the performance of MCA-SDR with that of MCA-EVR, PGA and sharpness-maximization autofocus algorithms. We used the focused image in Fig. 1a with a 2-D sinc-squared antenna pattern applied to it and $\mathrm{SNR}=60 \mathrm{~dB}$. The defocused image corrupted by a white phase error function is shown in Fig. 1 b. The MCA reconstruction using SDR is presented in Fig. 1c, and the reconstruction using EVR is shown in Fig. 1d. The PGA and sharpness-maximization reconstructions are shown 
(a)

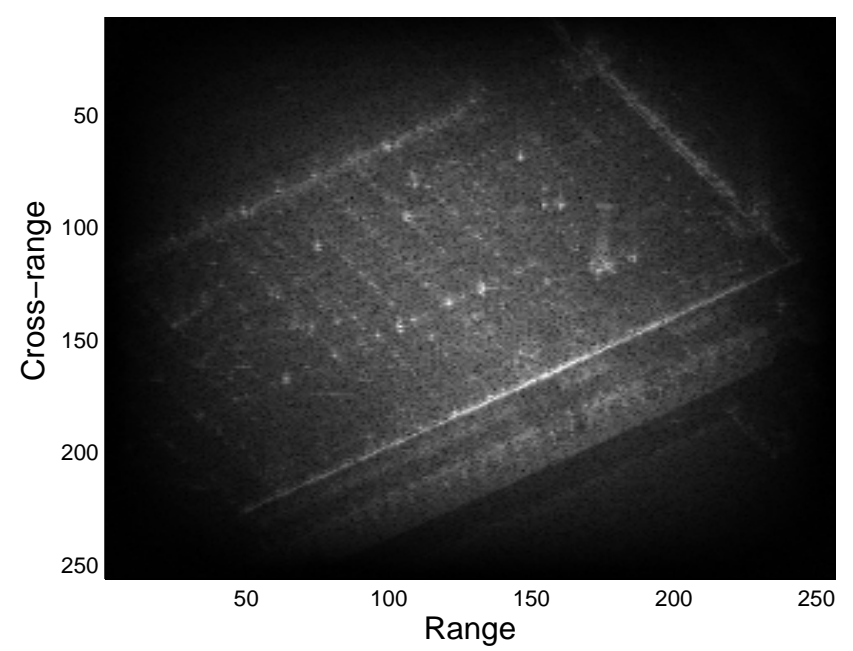

(c)

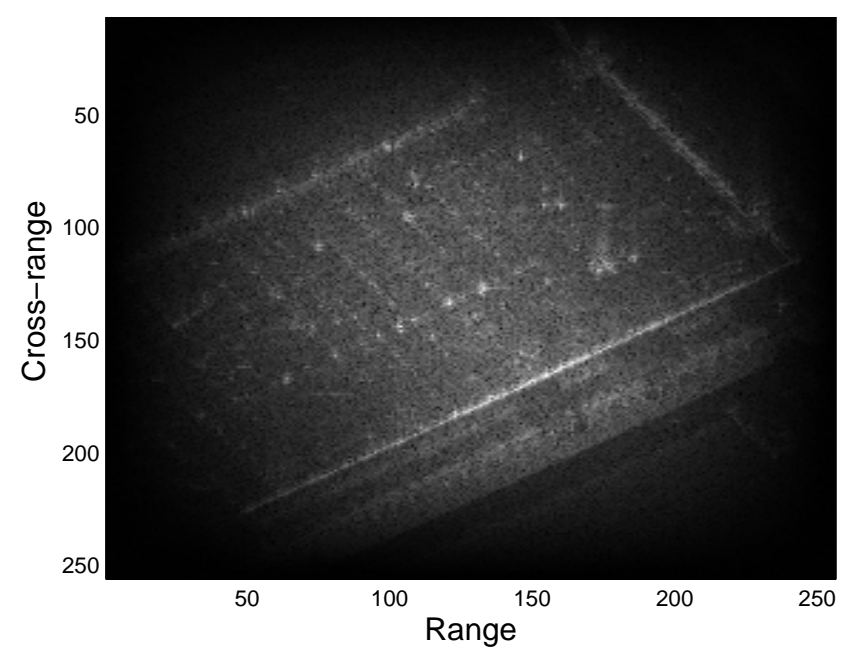

(e)

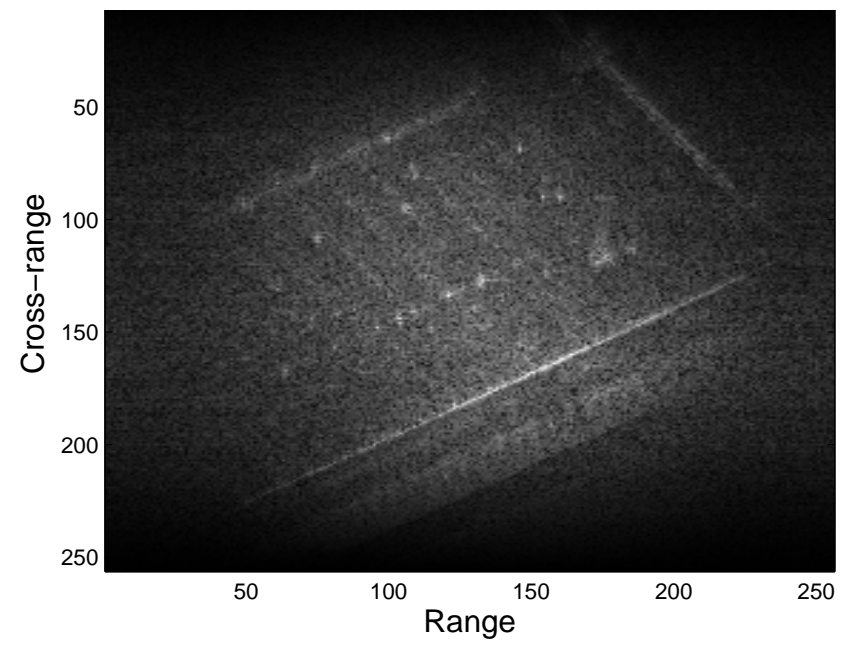

(b)

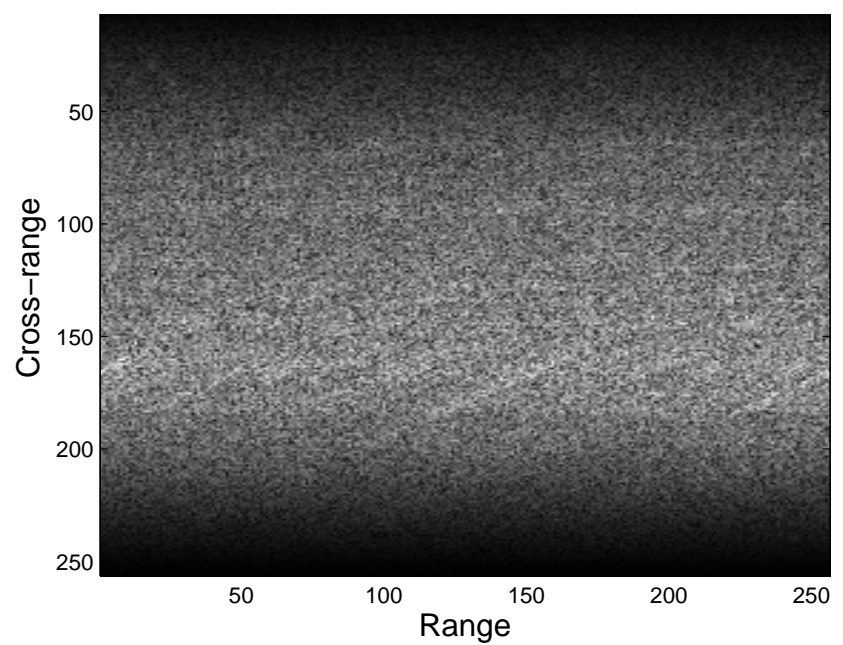

(d)

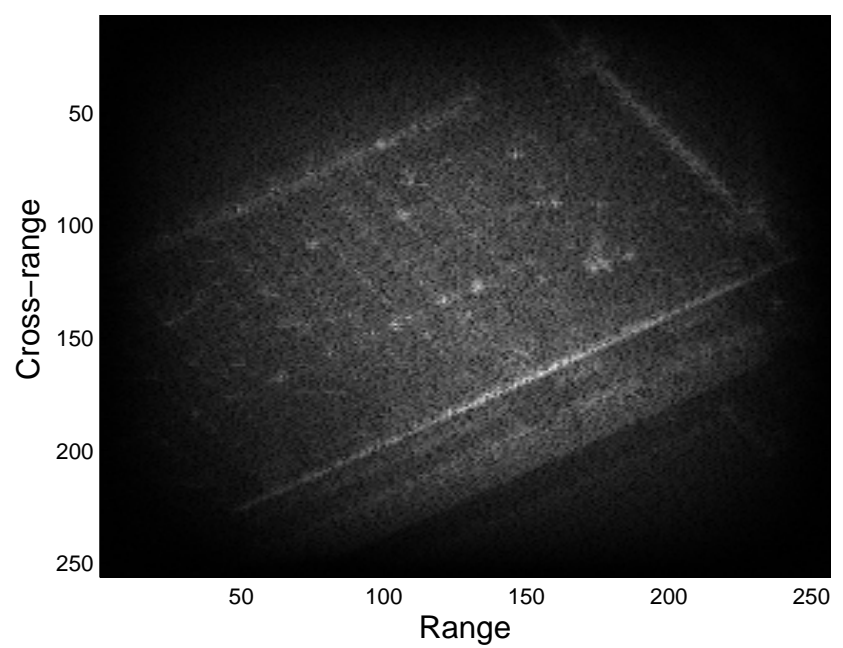

(f)

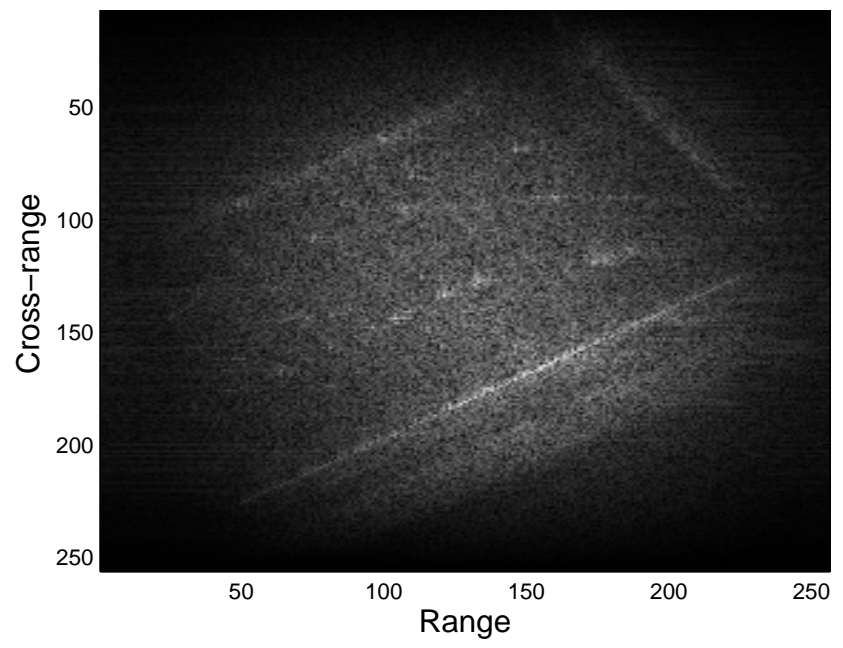

Fig. 1: Comparison of MCA-SDR with existing autofocus approaches: (a) perfectly focused image with 2-D sinc-squared antenna pattern applied and $\mathrm{SNR}=60 \mathrm{~dB}$; (b) defocused image corrupted by a white phase error function; (c) MCA-SDR restoration $\left(\mathrm{SNR}_{\text {out }}=15.3527 \mathrm{~dB}\right)$; $(\mathrm{d}) \mathrm{MCA}-\mathrm{EVR}$ restoration $\left(\mathrm{SNR}_{\text {out }}=8.1809 \mathrm{~dB}\right)$; (e) $\mathrm{PGA}_{\text {restoration }}(\mathrm{SNR}$ out $=9.3092 \mathrm{~dB})$; (f) Sharpness-maximization restoration $\left(\mathrm{SNR}_{\text {out }}=5.8177 \mathrm{~dB}\right)$. 
(a)

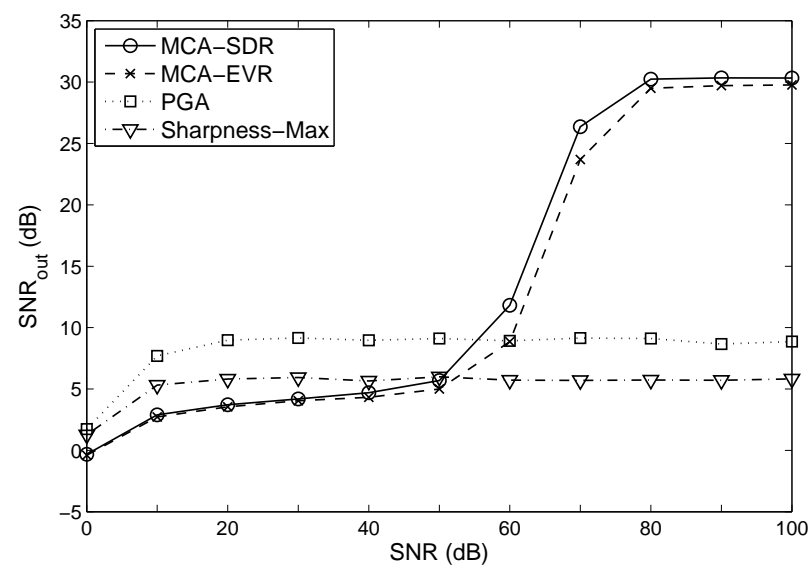

(b)

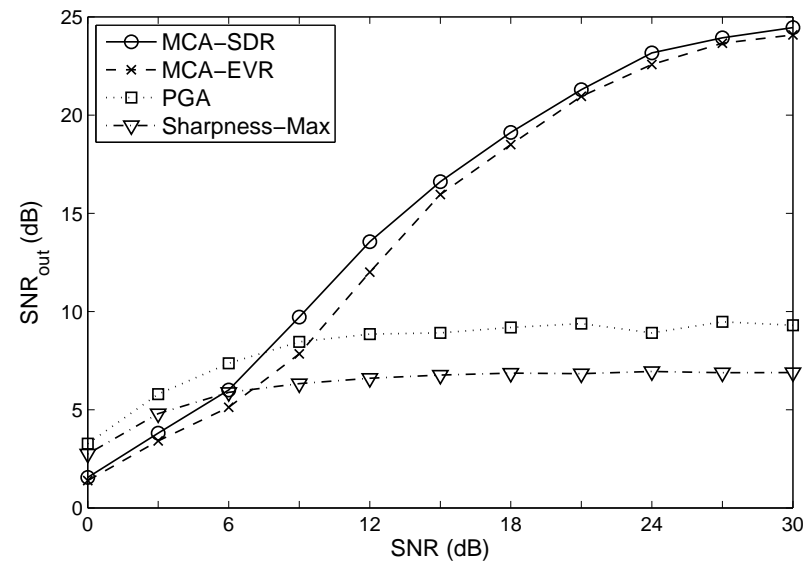

Fig. 2: Plots of the restoration quality metric $\mathrm{SNR}_{\text {out }}$ versus the input SNR for MCA-SDR, MCA-EVR, PGA and sharpness-maximization autofocus: (a) 2-D sinc-squared antenna pattern applied; (b) trapezoidal antenna pattern applied $\left(\gamma=10^{-4}\right)$.

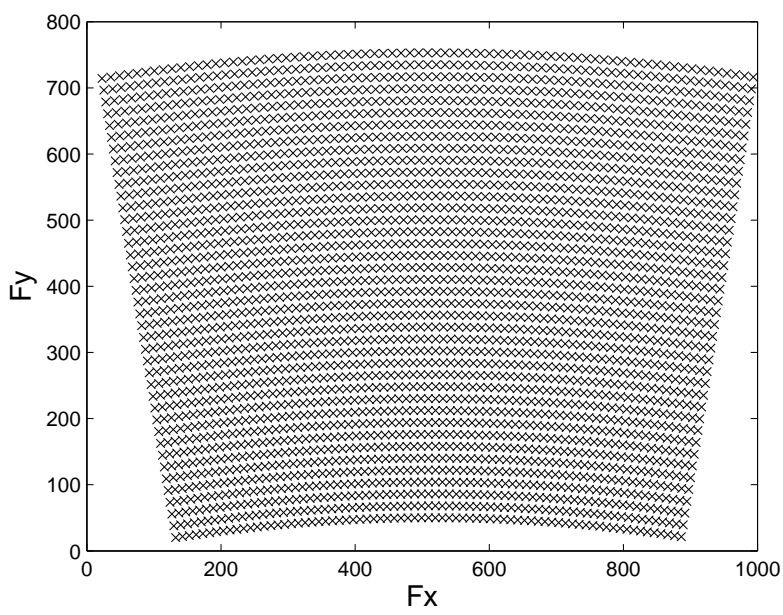

Fig. 3: Collected Fourier data pattern for wide-angle SAR (18 degree range of viewing angles). in Fig. 1e and Fig. 1f, respectively. A close inspection shows that the MCA-SDR reconstruction yields the best visual quality reconstruction.

To quantitatively evaluate the reconstruction we used the $\mathrm{SNR}_{\text {out }}$ metric defined as

$$
\mathrm{SNR}_{\text {out }}=20 \log _{10} \frac{\|\operatorname{vec}(g)\|_{2}}{\|(|\operatorname{vec}(g)|-|\operatorname{vec}(\hat{g})|)\|_{2}},
$$

where $\hat{g}$ is the reconstructed image. $\mathrm{SNR}_{\text {out }}$ measures the pixel-wise magnitude difference between the reconstructed image $\hat{g}$ and the perfectly focused image $g$. A higher $\mathrm{SNR}_{\text {out }}$ value corresponds roughly to a better restoration. The values of $\mathrm{SNR}_{\text {out }}$ for the above simulation are $15.3527 \mathrm{~dB}$ for MCASDR and $8.1809 \mathrm{~dB}, 9.3092 \mathrm{~dB}, 5.8177 \mathrm{~dB}$ for MCA-EVR, PGA and sharpness-maximization, respectively.

For this simulation we implemented SDR via the interiorpoint (IP) method [19]. The complexity of the IP method is on the order of $M^{3.5}$ which makes it computationally demanding for large $M$ as is the case for a real SAR system (usually in the thousands). However, there is a large body of research on large-scale SDP methods [27]. To give a rough idea of its computational cost, the run times for the above simulation on a HP Z200 (Quad 2.66 GHz) PC with 8GB of RAM were $14.1863,0.2936,2.2245$ and 67.4647 seconds for MCA-SDR, MCA-EVR, PGA and sharpness-maximization, respectively.

Next, we plotted SNR out $_{\text {v }}$ versus increasing SNR in Fig. 2a. Figure $2 \mathrm{~b}$ shows $\mathrm{SNR}_{\text {out }}$ versus $\mathrm{SNR}$ plotted for the same setup as in Fig. 2a but with a trapezoidal antenna pattern $(\gamma=$ $10^{-4}$ ) applied. From Fig. 2 we can see that MCA provides a better reconstruction than PGA and sharpness-maximization autofocus for high-SNR. Also, we can see that MCA-SDR is slightly superior to MCA-EVR.

In our second set of simulations, we adopted a SAR system operating across a wide range of look angles and compared FMCA-SDR with FMCA-EVR. In this simulation, we assumed an 18 degree range of look angles and used $M=1500$, $\mathrm{N}=800$ to generate the polar grid shown in Fig. 3 where $F_{x}$ and $F_{y}$ denote the coordinates of the 2-D Fourier space. The focused image is shown in Fig. 4a with a 2-D sinc-squared antenna pattern applied and $\mathrm{SNR}=80 \mathrm{~dB}$. The defocused image corrupted by a white phase error function is shown in Fig. $4 \mathrm{~b}$. The FMCA reconstruction using SDR is shown in Fig. $4 \mathrm{c}$ and the reconstruction using EVR is shown in Fig. 4d. It is easy to see the visual image enhancement due to the use of SDR. In order to demonstrate the effect of input SNR on restoration quality, Fig. 5 presents the output versus input SNRs for a 50 by 50 "toy" image collected on a polar grid with a 2 degree range of look angles. The toy image was generated randomly, with independent pixels having uniform magnitude and uniform phases. As expected, at high input SNR both EVR and SDR succeed in estimating the unknown phases, but the advantage of SDR is significant in cases with medium input SNR where the low-return region assumption is inexact.

We also report the results of an additional FMCA simulation conducted for a bistatic SAR system. We considered a bistatic SAR scenario where a moving transmitter and a moving receiver traverse a straight-line trajectory, perpendicular to each other. The corresponding Fourier data lies on a skewed 
(a)

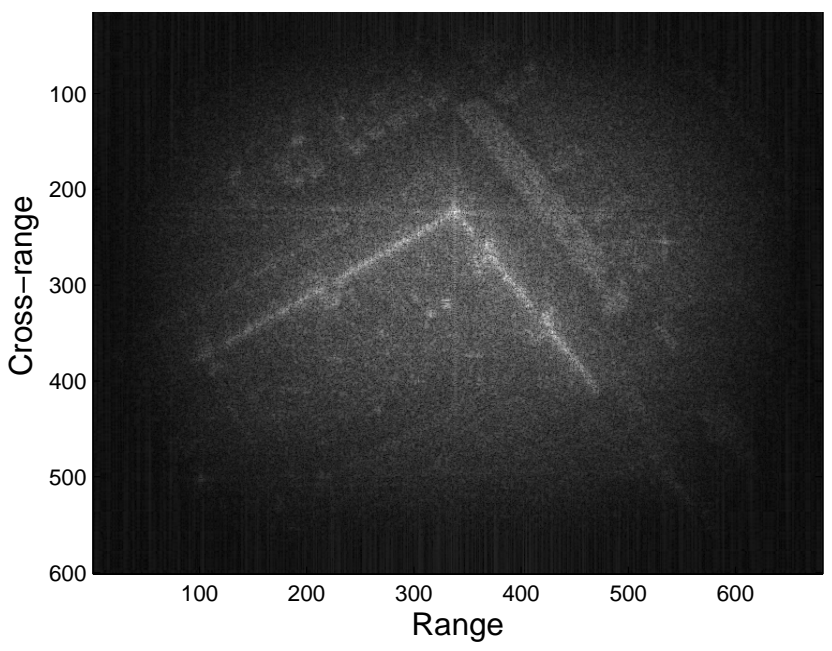

(c)

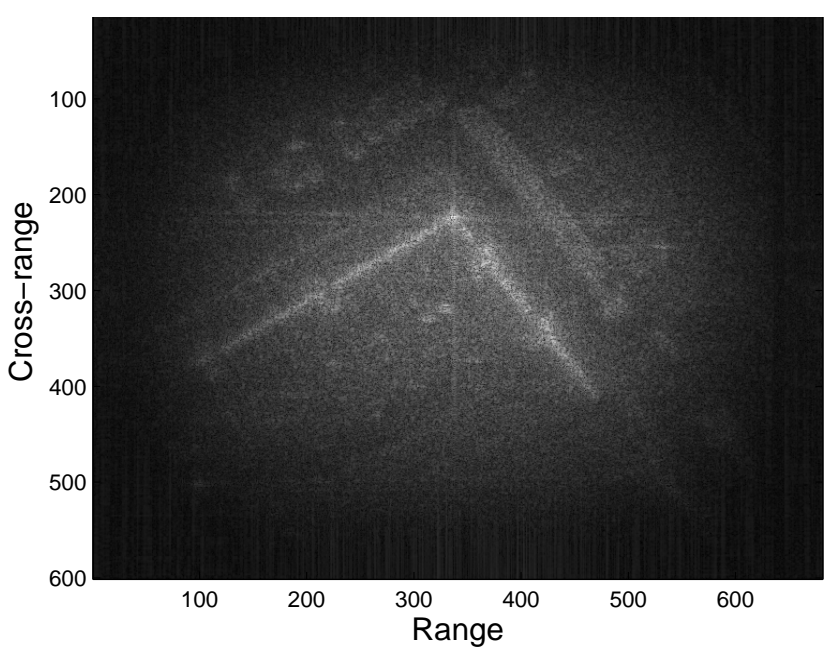

(b)

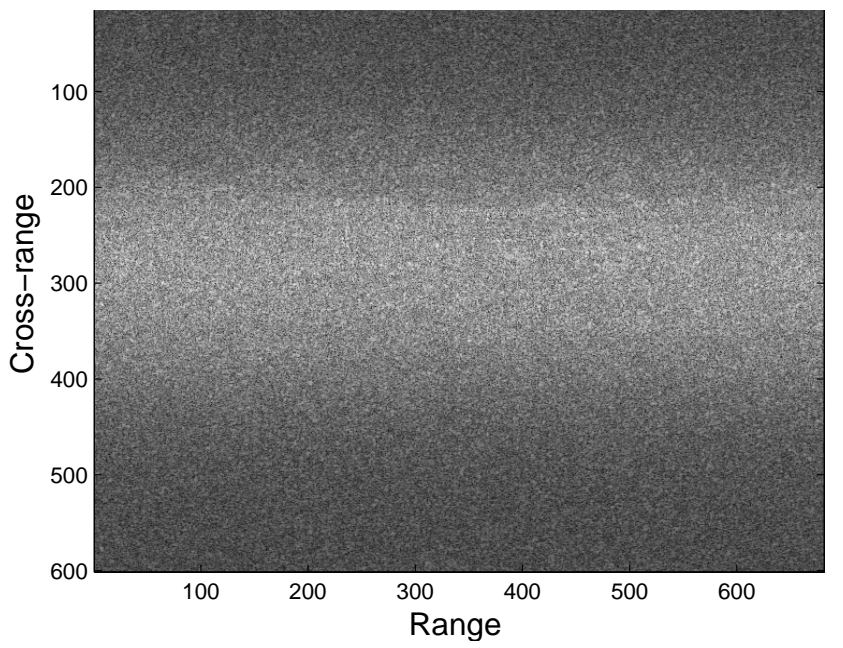

(d)

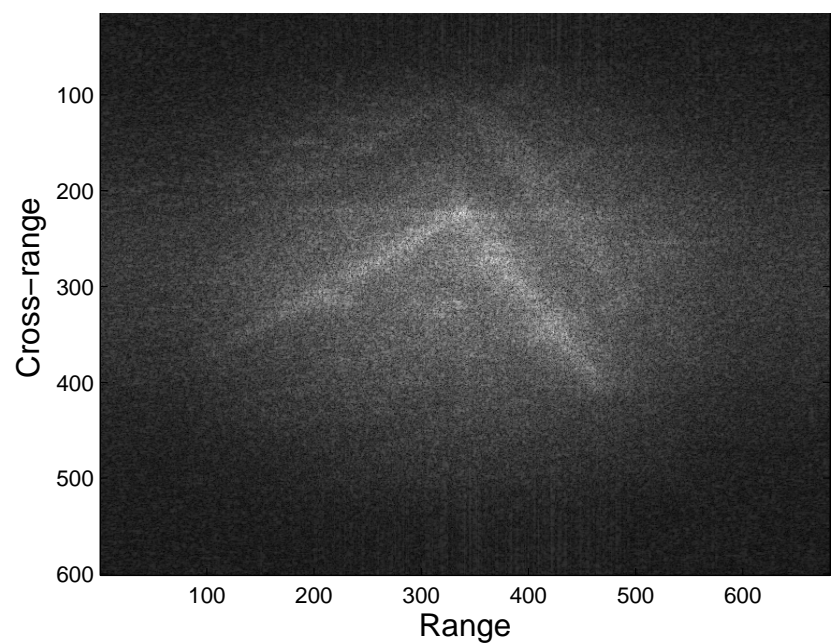

Fig. 4: Image restoration of FMCA-SDR and FMCA-EVR for wide-angle SAR autofocus: (a) perfectly focused image with 2-D sinc-squared antenna pattern applied and $\mathrm{SNR}=80 \mathrm{~dB}$; (b) defocused image produced by applying a white phase error function, (c) FMCA-SDR restoration $\left(\mathrm{SNR}_{\text {out }}=4.8718 \mathrm{~dB}\right)$; (d) FMCA-EVR restoration $\left(\mathrm{SNR}_{\text {out }}=3.1536 \mathrm{~dB}\right)$.

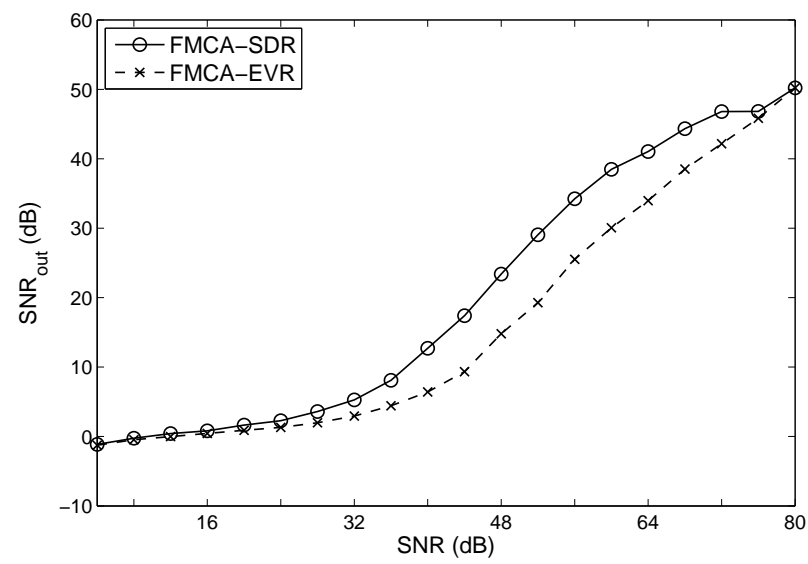

Fig. 5: Comparison of FMCA-SDR and FMCA-EVR image restoration, measured by $\mathrm{SNR}_{\text {out }}$.

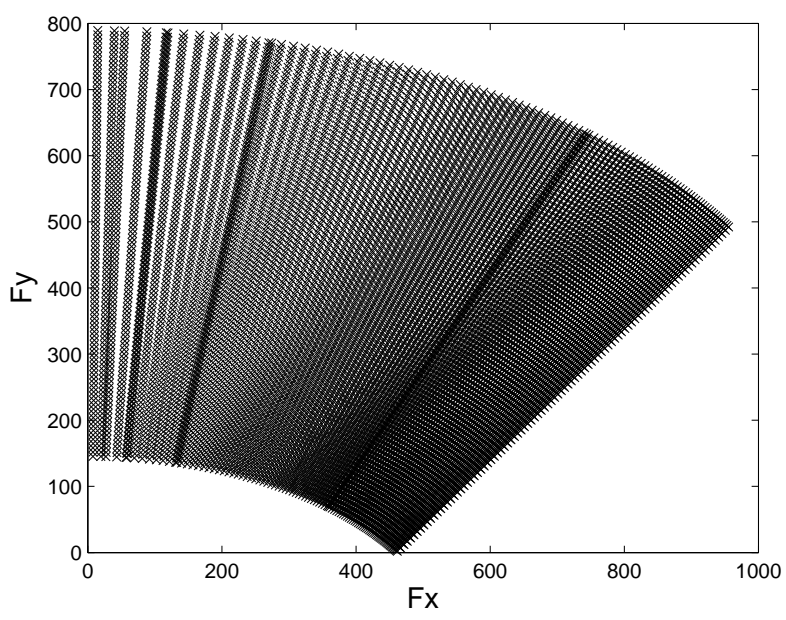

Fig. 6: Collected Fourier data pattern for bistatic SAR (moving transmitter and a moving receiver). 
(a)

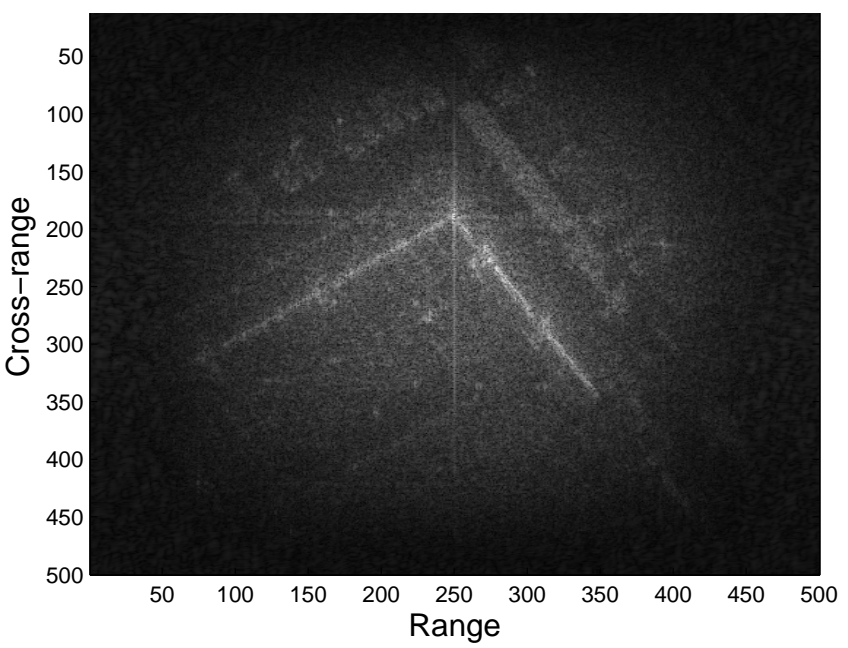

(c)

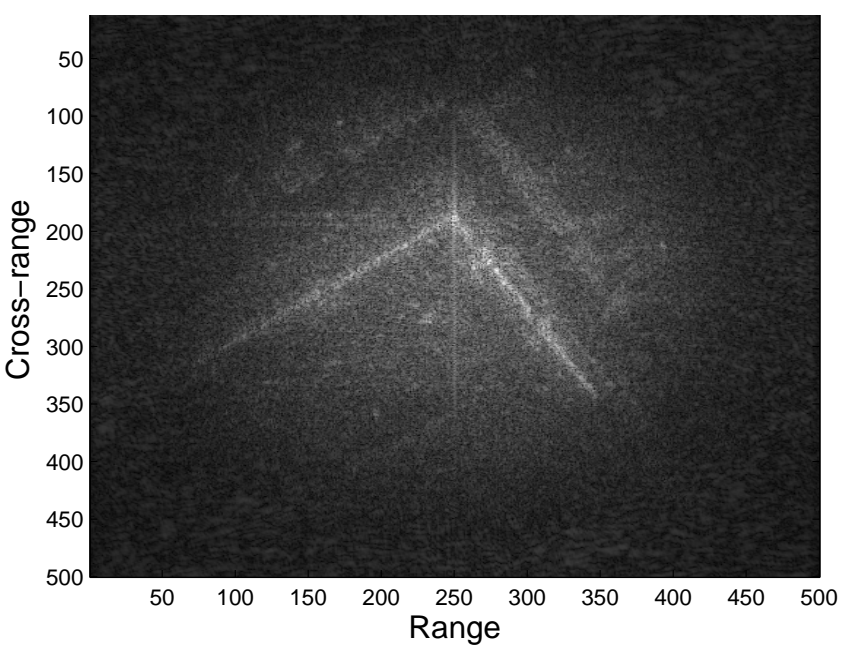

(b)

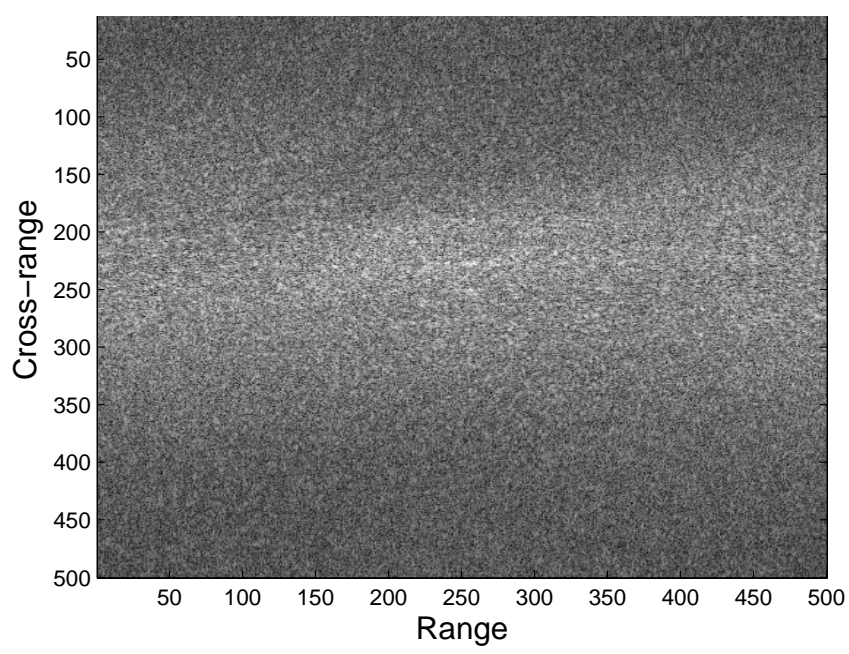

(d)

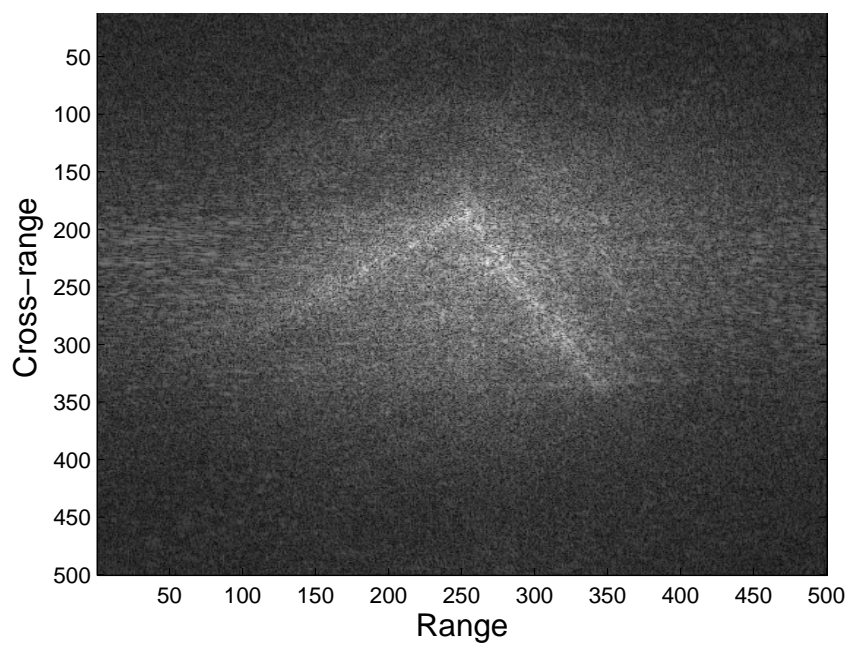

Fig. 7: Comparison of FMCA-SDR and FMCA-EVR for bistatic SAR autofocus: (a) perfectly focused image with 2-D sincsquared antenna pattern applied and SNR=50dB; (b) defocused image produced by applying a white phase error function; (c) FMCA-SDR restoration $\left(\mathrm{SNR}_{\text {out }}=4.6086 \mathrm{~dB}\right)$; $(\mathrm{d})$ FMCA-EVR restoration $\left(\mathrm{SNR}_{\text {out }}=2.1295 \mathrm{~dB}\right)$.

polar grid shown in Fig. 6 [28]. In this bistatic simulation we set $M=N=2000$. The focused image with a 2-D sincsquared antenna pattern applied to it and input SNR equal to $50 \mathrm{~dB}$ is shown in Fig. 7a. The defocused image, corrupted by a white phase error, is shown in Fig. 7b. The images restored by FMCA-SDR and FMCA-EVR are shown in Figs. 7c and $7 d$, respectively. Here too, the advantage of SDR over EVR is apparent.

The two FMCA simulations reported above correspond to a fairly large-scale SDP problem ( $M=1500$ for wide-angle simulation and $M=2000$ for bistatic simulation). For these two simulations, we implemented SDR via the bundle method [22]. To give a rough idea of its computational cost, the run time for the EVR simulation with Fig. 4a as input required less than 30 minutes on a HP Z200 (Quad 2.66 GHz) PC with 8GB of RAM, while SDR required about 24 hours to converge.

\section{Conclusion}

In this work, we proposed to use semidefinite relaxation (SDR) to improve two state-of-the-art SAR autofocus algorithms, namely Multichannel Autofocus (MCA) and Fourierdomain Multichannel Autofocus (FMCA). We first recognized that, although MCA and FMCA are developed based on different models and assumptions, they both attempt to find a solution to a constant modulus quadratic program (CMQP). CMQP is known to be NP-hard. MCA and FMCA both, either implicitly or explicitly, use eigenvalue relaxation to approximate the CMQP. We proposed to use SDR to approximate the CMQP arising in both algorithms. Experimental results showed that SDR provided promising image quality advantages over MCA and FMCA. Although solving autofocus problems using the new method is more computationally expensive, there may be crucial situations where it is imperative that an image be focused as well as possible, using computationally intensive off-line processing. 


\section{REFERENCES}

[1] C. V. Jakowatz, Jr., D. E. Wahl, P. H. Eichel, D. C. Ghiglia, and P. A. Thompson, Spotlight-Mode Synthetic Aperture Radar: A Signal Processing Approach. Boston: Kluwer Academic Publishers, 1996.

[2] D. C. Munson, Jr., J. D. OBrien, and W. K. Jenkins, "A tomographic formulation of spotlight-mode synthetic aperture radar," Proc. of the IEEE, vol. 71, pp. 917-925, August 1983.

[3] T. C. Calloway and G. Donohoe, "Subaperture Autofocus for Synthetic Aperture Radar," IEEE Transaction on Aerospace and Electronic Systems, vol. 30, no. 2, pp. 617-621, April 1994.

[4] G. A. Bendor and T. W. Gedra, "Single-Pass Fine-Resolution SAR Autofocus," Proc. of IEEE National Aerospace and Electronics Conference NAECON, Dayton, Ohio,, pp. 482-488, May 1983.

[5] T. J. Kragh, "Monotonic iterative algorithm for minimum-entropy autofocus," in Proc. Adaptive Sensor Array Processing Workshop, June 6-7 2006.

[6] X. Li, G. Liu, and J. Ni, "Autofocusing of ISAR images based on entropy minimization," IEEE Transactions on Aerospace and Electronic Systems, vol. 35, pp. 1240-1252, 1999.

[7] J. R. Fienup and J. J. Miller, "Aberration correction by maximizing generalized sharpness metrics," Optical Society of America, 2003.

[8] P. H. Eichel, D. C. Ghiglia, C. V. Jakowatz, Jr., and D. E. Wahl, "Phase-gradient autofocus for SAR phase correction: explanation and demonstration of algorithmic steps," in Proc. of the Digital Signal Processing Workshop at Starved Rock State Park, IL. IEEE, New York, 1992, pp. 6.6.1-6.6.2.

[9] R. L. Morrison, Jr., M. N. Do, and D. C. Munson, Jr., "MCA: A multichannel approach to SAR autofocus," IEEE Transactions on Image Processing, vol. 18, pp. 40 - 853, 2009.

[10] K. Liu and D. C. Munson, Jr., "Fourier-domain multichannel autofocus for synthetic aperture radar," Proc. 42nd Asilomar Conference on Signals, Systems and Computers, 2008, pp. 848 - 852, Oct 2008.

[11] Z. Luo and W. Yu, "An introduction to convex optimization for communications and signal processing," IEEE Journal on Selected Areas in Communications, vol. 24, pp. 1426-1438, 2006.

[12] W. Ma, P. Ching, and Z. Ding, "Semidefinite relaxation based multiuser detection for M-ary PSK multiuser systems," IEEE Journal on Selected Areas in Communications, vol. 52, pp. 2862- 2872, 2004.

[13] M. X. Goemans and D. P. Williamson, "Improved approximation algorithms for maximum cut and satisfiability problem using semi-definite programming," J.ACM, vol. 42, pp. 1115-1145, 1995.

[14] A. Wiesel, Y. Eldar, and S. Shitz, "Semidefinite relaxation for detection of 16-QAM signaling in MIMO channels," IEEE Signal Processing Letters, vol. 12, no. 9, pp. 653-656, 2005.

[15] S. Zhang and Y. Huang, "Complex quadratic optimization and semidefinite programming," SIAM Journal on Optimization, vol. 16, p. 871V890, 2006.

[16] M. So, J. Zhang, and Y. Ye, "On approximating complex quadratic optimization problems via semidefinite programming relaxations," Mathematical Programming, vol. 110, no. 1, pp. 93-110, Jun, 2007.

[17] A. Singer, "Angular synchronization by eigenvectors and semidefinite programming," Applied and Computational Harmonic Analysis, vol. In Press, Corrected Proof, 2010.

[18] K. Liu, A. Wiesel, and D. C. Munson, Jr., "Synthetic aperture radar autofocus via semidefinite relaxation," Proc. IEEE International Conference on Acoustics, Speech and Signal Processing, 2010, pp. 1342 1345, March 2010.

[19] S. Boyd and L. Vandenberghe, Convex Optimization. Cambridge University Press, March 2004.

[20] L. Vandenberghe and S. Boyd, "Semidefinite programming," SIAM Review, vol. 38, no. 1, pp. 49-95, 1996.

[21] J. Sturm, "Using SeDuMi 1.02, a MATLAB toolbox for optimization over symmetric cones," Optimization Methods and Software, pp. 625$653,1999$.

[22] C. Helmberg and F. Rendl, "A spectral bundle method for semidefinite programming," SIAM Journal on Optimization, vol. 10, pp. 673-696, 1997.

[23] C. Helmberg, "A cutting plane algorithm for large scale semidefinite relaxations," In The sharpest cut, MPS/SIAM Ser. Optim, Tech. Rep., 2001.

[24] D. C. Munson, Jr. and J. L. Sanz, "Image reconstruction from frequencyoffset fourier data," Proc. of the IEEE, vol. 72, no. 6, pp. 661-669, June 1984.

[25] H. Cho and D. C. Munson, Jr., "Overcoming polar-format issues in multichannel SAR Autofocus," Proc. 42nd Asilomar Conference on Signals, Systems and Computers, pp. 523-527, 2008.
[26] Sandia National Laboratory. Public SAR database. [Online]. Available: http://www.sandia.gov/radar/sar-data.html

[27] R. Monteiro, "First- and second-order methods for semidefinite programming," Mathematical Programming, vol. 97, pp. 209-244, 2003.

[28] O. Arikan and D. C. Munson, Jr., "A tomographic formulation of bistatic synthetic aperture radar," Intern. Conf. on Advances in Communication and Control Systems, pp. 289-302, 1988 Baton Rouge, La.,. 